\title{
On the influence of ram-pressure stripping on the star formation of simulated spiral galaxies
}

\author{
T. Kronberger, W. Kapferer, C. Ferrari, S. Unterguggenberger, and S. Schindler
}

\author{
Institute for Astro- and Particle Physics, University of Innsbruck, Technikerstr. 25, 6020 Innsbruck, Austria \\ e-mail: Thomas.Kronberger@uibk.ac.at
}

Received 23 October 2007 / Accepted 24 January 2008

ABSTRACT

\begin{abstract}
Aims. We investigate the influence of ram-pressure stripping on the star formation and the mass distribution in simulated spiral galaxies. Special emphasis is put on the question of where the newly formed stars are located. The stripping radius from the simulation is compared to analytical estimates.

Methods. Disc galaxies are modelled in combined $N$-body/hydrodynamic simulations (GADGET-2) with prescriptions for cooling, star formation, stellar feedback, and galactic winds. These model galaxies move through a constant density and temperature gas, which has parameters comparable to the intra-cluster medium (ICM) in the outskirts of a galaxy cluster $\left(T=3 \mathrm{keV} \approx 3.6 \times 10^{7} \mathrm{~K}\right.$ and $\left.\rho=10^{-28} \mathrm{~g} / \mathrm{cm}^{3}\right)$. With this numerical setup we analyse the influence of ram-pressure stripping on the star formation rate of the model galaxy.

Results. We find that the star formation rate is significantly enhanced by the ram-pressure effect (up to a factor of 3). Stars form in the compressed central region of the galaxy, as well as in the stripped gas behind the galaxy. Newly formed stars can be found up to hundred kpc behind the disc, forming structures with sizes of roughly $1 \mathrm{kpc}$ in diameter and with masses of up to $10^{7} M_{\odot}$. As they do not possess a dark matter halo due to their formation history, we name them "stripped baryonic dwarf" galaxies. We also find that the analytical estimate for the stripping radius from a Gunn \& Gott (1972) criterion agrees well with the numerical value from the simulation. Like in former investigations, edge-on systems lose less gas than face-on systems, and the resulting spatial distribution of the gas and the newly formed stars is different.
\end{abstract}

Key words. galaxies: interactions - galaxies: intergalactic medium - galaxies: stellar content - methods: numerical

\section{Introduction}

Many issues of galaxy formation and evolution can be understood within the framework of the successful cold dark matter (CDM) theory. But besides the hierarchical assembly of galaxies via mergers, other interaction phenomena are important in the cluster environment. Different types of such interactions are discussed: galactic winds, ram-pressure stripping, galaxygalaxy interactions and outflows from active galactic nuclei (AGN). Most of these processes can occur, with some principal differences, also in the field environment. Ram-pressure stripping, however, is uniquely linked to the presence of a surrounding medium that acts by its ram pressure. The space between the galaxies in a galaxy cluster is filled with a hot $\left(\sim 10^{8} \mathrm{~K}\right)$, thin $\left(\sim 10^{3}\right.$ ions $\left./ \mathrm{m}^{3}\right)$ plasma, the so called intra-cluster medium (ICM). Theoretically, the interaction between galaxies in the cluster and the ICM was already described by Gunn \& Gott (1972). They argued that galaxies lose material if the force due to ram-pressure stripping exceeds the restoring gravitational force of the galaxy. As a consequence, a stripping radius in ram-pressure affected galaxies can be defined, outside which the inter-stellar medium (ISM) cannot be prevented from being stripped by the galactic gravitational potential. Another analytical estimate was proposed by Mori \& Burkert (2000) where the thermal ISM pressure is compared with the external ram pressure. Later it was also found observationally that ram pressure acts on several galaxies in the Virgo cluster (e.g. Cayatte et al. 1990; Kenney et al. 2004; Vollmer et al. 2004) and in the Coma cluster (Bravo-Alfaro et al. 2000, 2001). Those galaxies, which are subject to ram-pressure stripping, lose parts of their inter-stellar medium to the ICM. As this gas has been enriched with heavy elements by the stars within the galaxy, the chemical abundance of the surrounding intra-cluster medium will be affected (Schindler et al. 2005; Domainko et al. 2006; Kapferer et al. 2007a). These heavy elements can then be found in X-ray observations.

Besides this treatment in cluster-scale simulations, several groups have already studied numerically the effect of rampressure stripping on individual galaxies. The stripping radius with respect to acting ram pressure and galaxy properties was investigated (e.g. Abadi et al. 1999; Vollmer et al. 2001; Roediger $\&$ Hensler 2005) and a reasonable agreement between the analytically estimated stripping radius and the stripping radius from numerical simulations was found. Further galaxy-scale numerical simulations for ram-pressure stripping were presented by Quilis et al. (2000), Mori \& Burkert (2000), Toniazzo \& Schindler (2001), Schulz \& Struck (2001), and Roediger \& Brüggen (2006, 2007). Very recently Jáchym et al. (2007) used an $N$-body/SPH code to investigate the influence of a timevarying ram pressure on spiral galaxies.

In contrast to these papers we focus on the effect of rampressure stripping on the star formation rate (SFR) of the galaxy, rather than on the mass loss. Nevertheless, for completeness we also analyse the mass loss and the resulting disc structure. The combined effects of ram-pressure stripping and tidal interactions on simulated cluster spirals is investigated in detail in Kapferer et al. (2007b). 
This paper is organised as follows: in Sect. 2 we present the numerical method and the simulation setup used for this work. In Sect. 3 the results are presented which are compared to observations in Sect. 4. We end with a summary of the main conclusions in Sect. 5.

\section{The numerical setup}

The simulations were carried out with the $N$-body/SPH code GADGET-2 developed by Springel (see Springel 2005, for details). This code treats the gas of the galaxies and the ICM hydrodynamically via smoothed particle hydrodynamics (SPH, Gingold \& Monaghan 1977; Lucy 1977) while the collisionless dynamics of the dark matter and the stellar component is calculated using an $N$-body technique. Prescriptions for cooling, star formation (SF), stellar feedback, and galactic winds are included as described in Springel \& Hernquist (2003). As we focus here on the effects of ram-pressure stripping on the star formation rate of the model galaxy, we summarise the main properties of the star formation model presented in Springel \& Hernquist (2003). In this formalism it is not attempted to spatially resolve star formation in the ISM but to describe star formation in a statistical manner. An SPH particle represents a certain region in the ISM with given values for the spatially averaged hydrodynamic quantities. Stars are assumed to form from cold gas clouds on a characteristic timescale $t_{\star}$ and a certain mass fraction $\beta$ is instantaneously released due to supernovae from massive stars (>8 $M_{\odot}$ ). The simple model for the time evolution of the stellar density $\rho_{\star}$ reads:

$\frac{\mathrm{d} \rho_{\star}}{\mathrm{d} t}=\frac{\rho_{\mathrm{c}}}{t_{\star}}-\beta \frac{\rho_{\mathrm{c}}}{t_{\star}}=(1-\beta) \frac{\rho_{\mathrm{c}}}{t_{\star}}$

where $\rho_{\mathrm{c}}$ is the density of the cold gas. As in Springel \& Hernquist (2003) we adopt $\beta=0.1$ assuming a Salpeter IMF with a slope of -1.35 in the limits of $0.1 M_{\odot}$ and $40 M_{\odot}$. In addition to the mass injection also energy is released to the ISM by the supernovae. This feedback energy heats the ambient gas and cold clouds can be evaporated inside hot bubbles of supernovae. All these assumptions lead to a self regulating star formation, where the growth of cold clouds is balanced by supernova feedback of the formed stars. The mass budget for the hot and cold gas densities ( $\rho_{\mathrm{h}}$ and $\rho_{\mathrm{c}}$, respectively) due to star formation, mass feedback, cloud evaporation and growth of clouds due to radiative cooling can then be written as

$\frac{\mathrm{d} \rho_{\mathrm{c}}}{\mathrm{d} t}=-\frac{\rho_{\mathrm{c}}}{t_{\star}}-A \beta \frac{\rho_{\mathrm{c}}}{t_{\star}}+\frac{1-f}{u_{\mathrm{h}}-u_{\mathrm{c}}} \Lambda_{\mathrm{net}}\left(\rho_{\mathrm{h}}, u_{\mathrm{h}}\right)$,

and

$\frac{\mathrm{d} \rho_{\mathrm{h}}}{\mathrm{d} t}=\beta \frac{\rho_{\mathrm{c}}}{t_{\star}}+A \beta \frac{\rho_{\mathrm{c}}}{t_{\star}}-\frac{1-f}{u_{\mathrm{h}}-u_{\mathrm{c}}} \Lambda_{\text {net }}\left(\rho_{\mathrm{h}}, u_{\mathrm{h}}\right)$

where $f$ represents a factor to differentiate between ordinary cooling $(f=1)$ and thermal instability $(f=0), \Lambda_{\text {net }}$ is the cooling function (Katz et al. 1996), and $A$ is the efficiency of the evaporation process. The onset of the thermal instability and hence of the star formation is set by a simple density threshold criterion, motivated by observations (Kennicutt 1989). Thus $f=0$ for densities larger than a certain threshold density $\rho_{\text {th }}$ and otherwise $f=1$.

The mass loss of the galaxy due to galactic winds, $\dot{M}_{\mathrm{w}}$, is assumed to be proportional to the star formation rate $\Psi_{\mathrm{SFR}}$, i.e. $\dot{M}_{\mathrm{w}}=\eta \Psi_{\mathrm{SFR}}$ with $\eta=2$, consistent with the observations of Martin (1999). Additionally, the wind contains a fixed fraction $\chi$
Table 1. Particle numbers and mass resolution used for the model galaxy.

\begin{tabular}{ccc}
\hline \hline & Particle number & $\begin{array}{c}\text { Mass resolution } \\
{\left[M_{\odot} / \text { particle }\right]}\end{array}$ \\
\hline Dark matter halo & 300000 & $3.5 \times 10^{6}$ \\
Disk collisionless & 200000 & $1.0 \times 10^{5}$ \\
Gas in disk & 200000 & $3.4 \times 10^{4}$ \\
ICM & 1000000 & $1.4 \times 10^{6}$ \\
\hline
\end{tabular}

of the supernova energy, which is assumed to be $\chi=0.25$. For further details and a description of the numerical implementation of this model we refer to Springel \& Hernquist (2003). This wind model is a purely phenomenological one, that lacks a physical foundation. Nevertheless, the model is constrained by observational data, as far as possible, and should therefore be able to give plausible results.

The initial conditions were built according to Springel et al. (2005), based on the analytical work of Mo et al. (1998). The model galaxy, which we use, represents a Milky-Way type spiral galaxy. The total mass of the model galaxy is $1.09 \times 10^{12} M_{\odot}$, where the initial total gas mass is $6.8 \times 10^{9} M_{\odot}$. Compared to previous work (Kapferer et al. 2005, 2006a; Kronberger et al. 2006, 2007), where we used a similar sized galaxy, we have significantly increased the number of particles, increasing the mass resolution of the simulation. Important numerical quantities, such as the particle numbers, are summarized in Table 1. For the intra-cluster medium we use one million SPH particles, which we distribute homogeneously over a volume of $1 \mathrm{Mpc}^{3}$ with a mass density of $1 \times 10^{-28} \mathrm{~g} / \mathrm{cm}^{3}$ and a constant temperature of $3 \mathrm{keV}\left(\sim 3.6 \times 10^{7} \mathrm{~K}\right)$. Such a simplified ICM distribution allows to study the effects of ram-pressure stripping in a clean way, i.e. with as little degeneracies of different effects as possible. Additional effects from varying density and temperature in the ambient medium will be investigated in an upcoming work.

Starting from these initial conditions we calculate the evolution of the model galaxy moving with a constant velocity of $1000 \mathrm{~km} \mathrm{~s}^{-1}$ through the ambient medium for 1 Gyr. A galaxy typically will not move through a homogeneous ICM for 1 Gyr. Therefore, to keep our results comparable to observations of galaxies in the outskirts of galaxy clusters, we study primarily the first few hundred Myr. We calculated two different interaction geometries, one in which the galaxy flies face-on through the ambient medium and one in which the ram-pressure acts edge-on. In order to avoid numerical artifacts we use for the investigation of ram-pressure stripping a model galaxy after $2 \mathrm{Gyr}$ of isolated evolution. After that period of time self-regulated star formation is present and the gas distribution has settled from the simple analytic initial condition to an equilibrium distribution with a spiral structure.

To study possible resolution effects, originating from the different mass resolution of ICM and ISM particles, we carry out a simulation with $10^{7} \mathrm{ICM}$ particles. We found no significant differences to the lower resolution runs concerning the results presented below for the star formation rate or the mass distribution.

The SPH method has some intrinsic shortcomings that might affect the results to some extent. One is the resolution of instabilities and turbulence (e.g. Agertz et al. 2007). The shear flows present in systems affected by ram-pressure stripping can lead to the development of Kelvin-Helmholtz instabilities (e.g. Roediger et al. 2006), which are not resolved in our setup. Instabilities should induce turbulence and fragmentation and therefore enhance star formation which is consistent with our 
result. A similar study with a Eulerian code is a desirable future work to investigate the importance of hydrodynamic instabilities in this issue.

\section{Results}

In the following we will first analyse the mass loss of the model galaxy due to ram-pressure stripping. Then we will present the results concerning the star formation rates and the distribution of the newly formed stars, within the adopted hydrodynamic and star-formation model.

\subsection{The stripping radius and the mass distribution inside the stripped galaxy}

The Gunn \& Gott (1972) criterion, according to which galaxies lose material if the force due to ram-pressure stripping exceeds the restoring gravitational force of the galaxy, implies the existence of a stripping radius. This is the distance from the galactic centre, outside which the galactic gravitational potential cannot prevent the ISM from being stripped. The position of this stripping radius depends on the mass distribution in the galaxy and the external pressure exerted by the ICM. Assuming a double exponential density distribution of the stars and the gas in the disc, the expression for the stripping radius, $R_{\text {strip }}$, reads (Domainko et al. 2006):

$\frac{R_{\text {strip }}}{R_{0}}=0.5 \times \ln \left(\frac{G M_{\mathrm{star}} M_{\mathrm{gas}}}{v_{\mathrm{gal}}^{2} \rho_{\mathrm{ICM}} 2 \pi R_{0}^{4}}\right)$.

Here $M_{\text {star }}$ and $M_{\text {gas }}$ are the mass of the stellar and gaseous disc, respectively, $v_{\text {gal }}$ denotes the velocity of the galaxy relative to the ICM, $\rho_{\mathrm{ICM}}$ is the density of the surrounding ICM, and $R_{0}$ is the radial scale length of the disc. The validity of this approximation was already shown using numerical simulations and by comparison with Virgo cluster galaxies by Abadi et al. (1999).

We also find a fair agreement between the analytical value and the stripping radius in our simulation. From Eq. (4) we calculate a theoretical value of $13.2 \mathrm{kpc}$. In Fig. 1 the gas density profile of the stripped model galaxy (dashed line), which moves face-on through the ICM, is compared to the isolated one (solid line) at the same evolutionary time. The ISM distribution is shown after $100 \mathrm{Myr}$ of ram pressure acting. There is a clear drop in the density at a radius of $r \sim 13 \mathrm{kpc}$ visible, which corresponds well to the analytically derived stripping radius. At radii larger than $15 \mathrm{kpc}$ the gas is almost completely stripped. While in the simple theoretical model the gas distribution inside the stripping radius is not affected, it adjusts in the hydrodynamic simulation to the modified mass distribution and pressure gradients and changes due to the mass loss caused by galactic winds. At radii $r<10 \mathrm{kpc}$ the gas density lies below the density of the isolated galaxy, while near to the stripping radius the density increases with respect to the isolated one and forms a peak. In the central regions of the ram-pressure affected disc the starformation rate is enhanced (see Sect. 3.2), which depletes the gas reservoir there due to the conversion of gas to stars and a subsequently enhanced galactic wind. Once established this density distribution remains qualitatively in this shape, while quantitatively it changes with time due to the acting galactic wind.

The total gas mass loss due to ram-pressure stripping and galactic winds is shown in Fig. 2 for the first 500 Myr. For this figure we take only the gravitationally unbound gas into account, i.e. the gas which has a positive total energy. Note that this mass loss is a superposition of two processes, ram-pressure

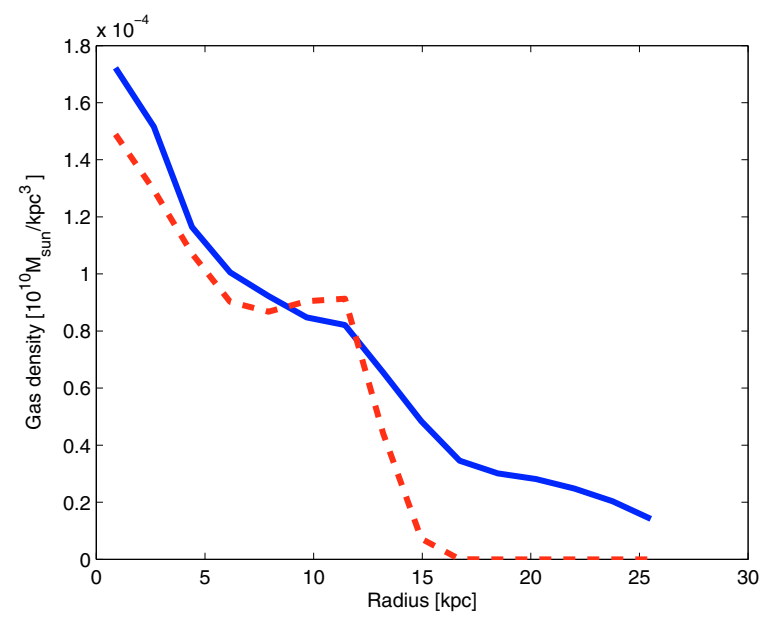

Fig. 1. Radial gas density profile for the isolated model galaxy (solid line) and the stripped model galaxy (dashed line), which moves faceon through the ICM. The distribution is shown after $100 \mathrm{Myr}$ of ram pressure acting. A drop of the ISM density at $\sim 13 \mathrm{kpc}$ is clearly visible.

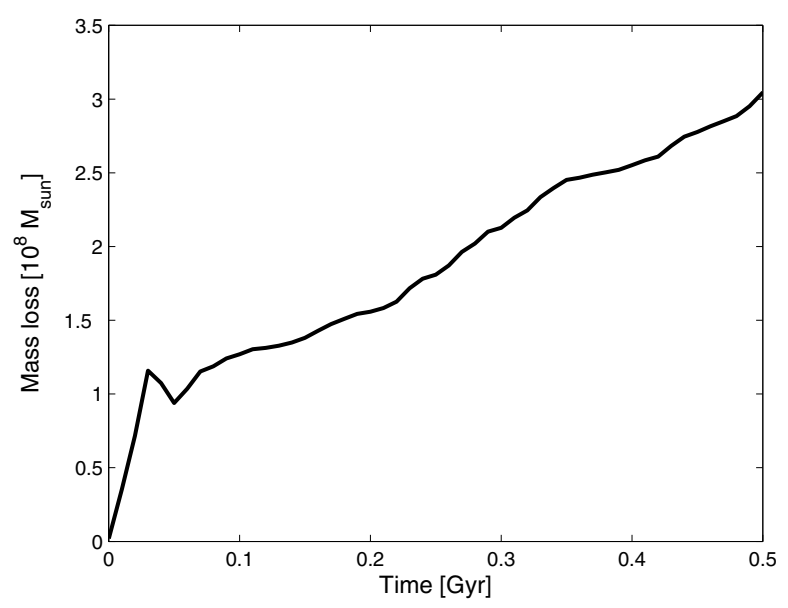

Fig. 2. Integrated total mass loss due to ram-pressure stripping and galactic winds of the stripped model galaxy, which moves face-on through the ICM. Note that only the gravitationally unbound gas is plotted. Even a decrease of the mass loss is possible, if gas enters, for example, the slipstream of the galaxy, as visible after $\approx 50 \mathrm{Myr}$.

stripping and galactic winds. There is an interplay between both effects, as ram-pressure stripping changes the star formation rate, which in turn changes the galactic wind mass loss. As many authors have already investigated the mass loss due to ram-pressure stripping and compared the numerical results to analytical estimates (e.g. Jáchym et al. 2007) we do not study the isolated effect of ram-pressure stripping here. However, we point out once more, that the stripping radius in our simulations is comparable to analytical estimates, as found by various authors. In the time after the stripping radius becomes clearly visible, galactic winds should be the dominating outflow mechanism. The mass loss then stays roughly constant over time. Note, however, that the ambient medium acts on the galactic wind by its pressure and that the mass of the gas actually lost by the galactic wind is hence smaller than expected from the star formation rate. Such a suppression of galactic winds has already been discussed in a different context by Schindler et al. (2005).

Additionally we investigated the spatial distribution of the stripped gas. We find that after $100 \mathrm{Myr} 13.7 \%\left(9.3 \times 10^{8} M_{\odot}\right)$ of the initial ISM mass is in the wake of the galaxy. We define 


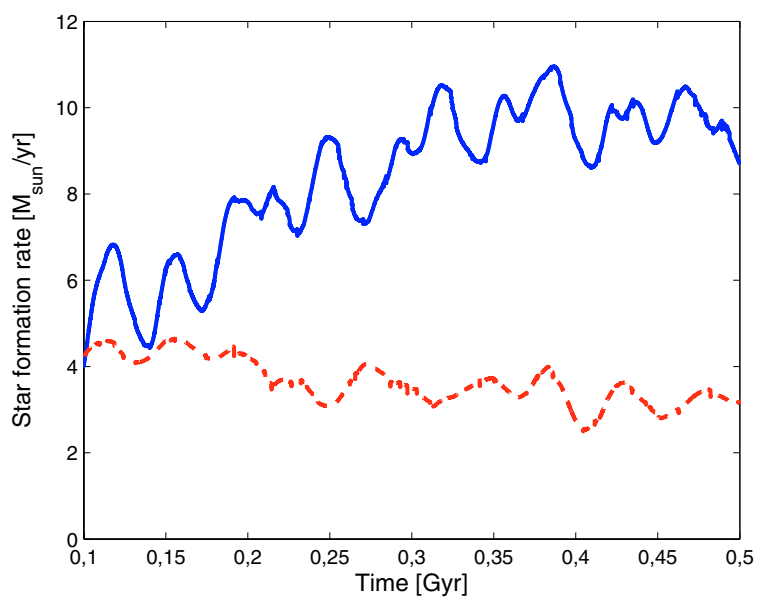

Fig. 3. Total star formation rate as a function of time for an isolated model galaxy (red, dashed line) and for a ram-pressure stripped model galaxy (blue, solid line), which moves face-on through the ICM. Note that the increase in the star-formation rate is the physically relevant information. The apparent oscillations are due to the numerical implementation of the star-formation model.

this to be the region more than 6 times the scale height away from the disc, which is for our model galaxy $1.3 \mathrm{kpc}$. This distance should be significantly larger than the half-light radius of the galaxy, and therefore observationally be considered as not belonging to the galaxy. After $200 \mathrm{Myr}$, this amount of stripped gas increases to $31.7 \%\left(2.2 \times 10^{9} M_{\odot}\right)$ of the initial ISM mass. However, material caught in the slipstream of the moving galaxy does not feel the ram pressure of the ICM and a significant proportion will fall back onto the disk. After $500 \mathrm{Myr}$ about $10 \%$ $\left(6.8 \times 10^{8} M_{\odot}\right)$ of the initial ISM mass are in the wake of the galaxy and gravitationally unbound.

We performed the same analysis for the case in which the model galaxy moves edge-on through the medium. In this case no clear stripping radius can be identified. The gaseous disc is compressed and distorted but the mass loss is lower than for the face-on galaxy. After $100 \mathrm{Myr}$ there is only $0.9 \%\left(6.1 \times 10^{7} \mathrm{M}_{\odot}\right)$ of the initial ISM mass in the wake of the galaxy. This fraction increases to $8.6 \%\left(5.8 \times 10^{8} M_{\odot}\right)$ after $200 \mathrm{Myr}$ and finally after 500 Myr $6.3 \%\left(4.3 \times 10^{8} M_{\odot}\right)$ of the initial ISM mass is in the wake of the galaxy and gravitationally unbound. Also in the edge-on case we find re-accretion in the slipstream of the galaxy.

\subsection{The impact on star formation}

There has been a lot of discussion whether ram-pressure stripping acting on a galaxy enhances or decreases the star formation rate (e.g. Evrard 1991; Fujita et al. 1999). With our simplified setup that studies the influence of a homogeneous medium, we find a clear enhancement, within the adopted star formation model. In Fig. 3 we plot the total star formation rate as a function of time for an isolated model galaxy and for a ram-pressure stripped model galaxy, which moves face-on through the ICM. Over 500 Myr the star formation rate is always significantly (up to a factor of 3) higher than in the isolated galaxy. Most of the new stars form in the disc, mainly in the compressed central region. However, star formation is also present in the stripped material in the wake of the galaxy. These stars are not gravitationally unbound but can spread over a large volume in the galactic halo. We find newly formed stars up to hundred kpc behind the plane of the disc. As these stars do not feel the ram pressure of

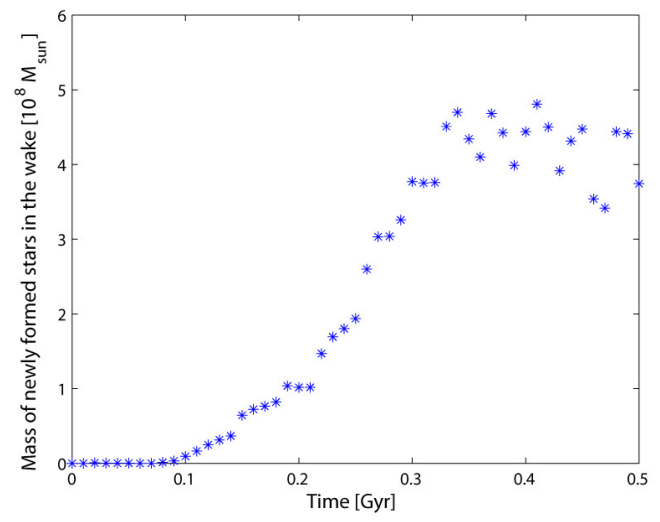

Fig. 4. Mass of stars formed in the wake of the model galaxy as a function of time. The ram pressure acts face-on. The scatter in the plot is a consequence of stars falling back into the gravitational potential towards the centre of the disc.

the surrounding ICM, they fall back into the gravitational potential towards the centre of the disc. The kinetic energy they gain hereby is sufficient that they partly cross the galactic plane and enter the space in front of the disc. In the ram-pressure affected galaxy about twice as many stars are formed as in the isolated galaxy in the time while ram-pressure stripping is acting. After 200 Myr of acting ram pressure the total mass of newly formed stars in this period is $1.02 \times 10^{8} M_{\odot}$ in the wake of the galaxy, while in the same time in the disc $9.5 \times 10^{8} M_{\odot}$ form. (The wake of the galaxy is defined, as before, to be more than 6 times the scale height away from the disc. The disc region itself extends, by our definition, to four times the stellar disc scale length in radial direction and twice the scale height in the direction perpendicular to the disc.) Note that these masses also depend on the chosen IMF, as this regulates the amount of massive stars that end instantaneously (i.e. in less than a typical numerical time step) as supernovae. Therefore the mass of the newly formed stars does not include the high-mass stars (>8 $M_{\odot}$ ). In Fig. 4 we show the mass of the newly formed stars as a function of time. The scatter in the plot is a consequence of stars falling back into the gravitational potential towards the centre of the disc.

The distribution of the gas and the newly formed stars is presented in Figs. 6 and 7 for the ram-pressure affected galaxy, which moves face-on through the ICM. Note that newly formed stars are here defined to be stellar particles formed after the beginning of the simulation. After $100 \mathrm{Myr}$ the stripping is already active and a stripping radius is visible. A wake, however, has not yet formed, and the star formation mainly takes place in the central part of the galaxy, where the gas is compressed by the ram pressure of the surrounding ICM. After $500 \mathrm{Myr}$ a wake has formed with dwarf galaxy sized sub-structures (diameter $\sim 1 \mathrm{kpc}$, masses up to $10^{7} M_{\odot}$ in gas and newly formed stars). These objects formed by initial compression and subsequent cooling of already slightly overdense regions in the disc (parts of spiral arms). This formation scenario is different from the gravitational collapse, as discussed for tidal dwarf galaxies in tidally interacting systems. Note that due to their formation history from stripped material, these structures do not have a dark matter halo. We therefore term these structures "stripped baryonic dwarf" galaxies. Most of these structures eventually fall back onto the galactic disc on timescales of $\leq 1 \mathrm{Gyr}$. In more realistic systems with a gravitational cluster potential and interactions with other galaxies the fate of these substructures might 


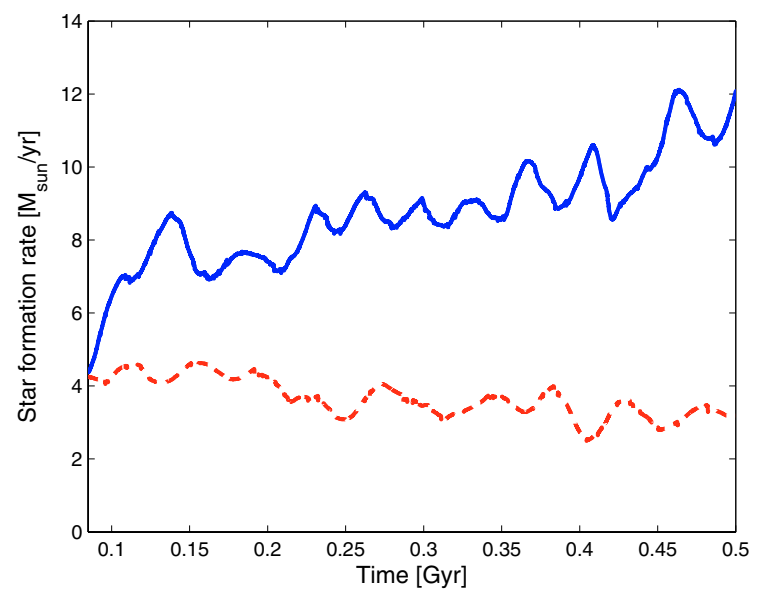

Fig. 5. Total star formation rate as a function of time for an isolated model galaxy (red, dashed line) and for a ram-pressure stripped model galaxy (blue, solid line), which moves edge-on through the ICM. Note that the increase in the star-formation rate is the physically relevant information. The apparent oscillations are due to the numerical implementation of the star-formation model.

be different. Especially the ram pressure of the ICM usually changes direction, as not all orbits are purely radial. Kapferer et al. (2007b) show that such structures also form when galaxy galaxy interactions take place in an ambient medium. They are then distributed over much larger distances compared to the case presented here. It has to be further investigated, whether these objects can then form virialised, long-term stable structures. In Fig. 7 we show gas and newly formed stars separately, in order to show their distribution more clearly. As stars are collisionless their distribution differs from that of the gas, the small substructures are, however, visible in both distributions. From the stripped gas only around $25 \%$ is heated to temperatures above $1 \times 10^{7} \mathrm{~K}$ after $500 \mathrm{Myr}$ of acting ram pressure, and hence visible in X-rays. Especially in the dense knots, where stars are formed, the temperature remains significantly lower.

The situation is very similar in the edge-on case, although the resulting mass distribution is different. In Fig. 5 the total star formation rate as a function of time is shown for an isolated model galaxy and for a ram-pressure stripped model galaxy, which moves edge-on through the ICM. The enhancement of the star formation rate is here even stronger than in the face-on case. These new stars are, however, mainly formed in the central region of the disc. The gas from the outskirts of the galactic disc is pushed to the central region, enhancing there significantly the density of the cold gas and hence the star formation rate.

From the results presented in this section we would therefore expect especially in galaxy cluster mergers an increased star formation activity in the merger region, because the galaxies encounter there a rapid rise of the external ram pressure. As a lot of gas from the galaxy is lost by ram-pressure stripping or converted to stars, the cold gas reservoir is constantly depleted. Hence, the star formation rate first increases and eventually decreases.

\section{Comparison of the results to observations}

An evolution of the cluster population with redshift has been pointed out since the late 70's. The fraction of star-forming and post-star-forming systems has been proven to significantly increase with $z$, going from $\geq 30 \%$ at $z \sim 0.3-0.5$ (Dressler et al. 1999) to $\sim 1-2 \%$ in the local Universe (e.g. Dressler 1987). In

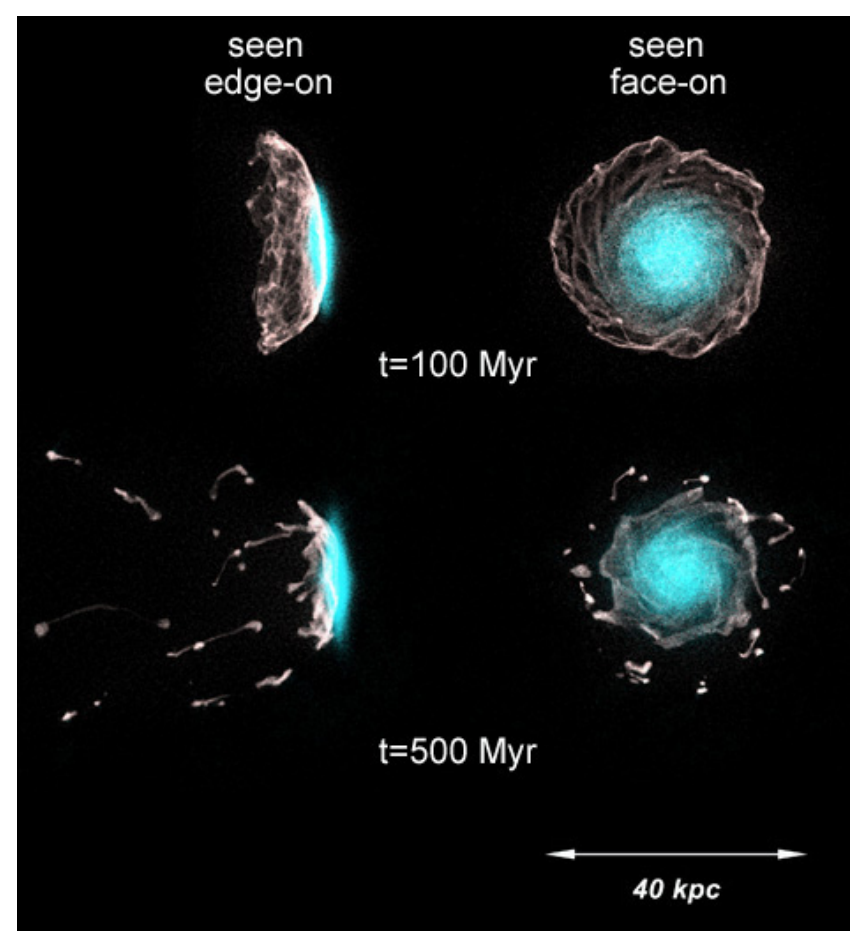

Fig. 6. Distribution of the gas (white) and the newly formed stars (turquoise) for a ram-pressure affected galaxy, which moves face-on through the ICM, after 100 Myr (top) and after 500 Myr (bottom), seen face-on and edge-on.

agreement with this tendency, in 1978 Butcher \& Oemler reported a strong evolution from bluer to redder colours in cluster galaxies, detecting an excess of blue objects at $z=0.5$ with respect to lower redshift systems ("Butcher \& Oemler effect"). In order to link the observed evolution of cluster members to the hierarchical growth of structures, we still need to understand the specific importance of each of the proposed physical mechanisms acting on the star formation activity and on the morphology of galaxies (i.e. galaxy mergers and interactions: e.g. Lavery \& Henry 1988 - ram-pressure stripping: Gunn \& Gott 1972 "harassment": e.g. Moore et al. 1998 - "strangulation" or "starvations": e.g. Larson et al. 1980).

Quantifying the evolution of SF in cluster members, however, is extremely difficult, since the fraction of star-forming objects varies from one cluster to another also in the same redshift range (see Poggianti 2004, and references therein). It is thus essential to study simultaneously the evolution of galaxies as a function of redshift and of the properties of their host cluster. Ferrari et al. (2005) have for instance proven that the fraction of star-forming and post-star-forming galaxies in the low redshift $(z \simeq 0.09)$ cluster A3921 is comparable to those measured at intermediate redshifts. A3921 is a merging system (Ferrari et al. 2005; Belsole et al. 2005), and a detailed comparison of optical and radio observations revealed that most of the star-forming galaxies are located in the collision region of the two interacting sub-structures (Ferrari et al. 2005, 2006). This suggests that the high fraction of star-forming objects in A3921 is very likely related to its dynamical state.

Other analyses agree with this result, suggesting that SF is either triggered by cluster mergers (e.g. Coma: Caldwell et al. 1993 - A3562: Bardelli et al. 1998; Miller 2005 - A2125: Dwarakanath \& Owen 1999; Owen et al. 2005 - A2255: Miller \& Owen 2003), or quenched after a starburst phase, leaving 

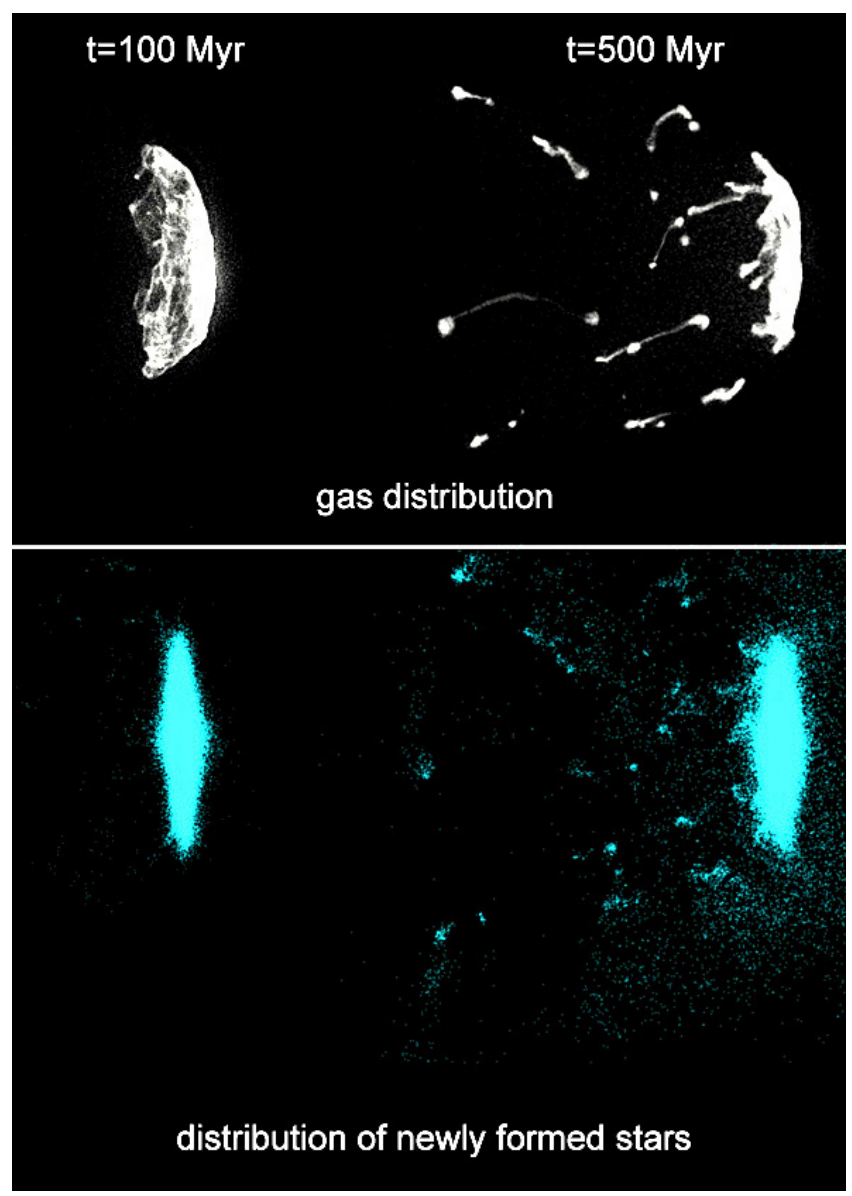

Fig. 7. Distribution of the gas (white, top) and the newly formed stars (turquoise, bottom) seen edge-on for a ram-pressure affected galaxy, which moves face-on through the ICM, after $100 \mathrm{Myr}$ (first column) and after $500 \mathrm{Myr}$ (second column).

young and strong poststarburst galaxies (e.g. Coma: Poggianti et al. 2004). Other studies (e.g. A168: Tomita et al. 1996 A3528: Bardelli et al. 2001) did not show any correlation between cluster mergers and on-going SF. All these results suggest that cluster collisions are able to affect the internal properties of galaxies, and in particular their star formation history, possibly inducing an initial starburst phase, that consumes most of the gas in the galaxy, creating a subsequent decrease in the star formation rate of cluster members. Note that, among the previously mentioned clusters, those systems that are observed $\sim 0.2$ Gyr before/after the closest cores encounter show increased SF (e.g. A3921: Kapferer et al. 2006b - A2125: Miller et al. 2005 - A2255: Sakelliou \& Ponman 2006), contrarily to A168, that instead is as post-merger cluster observed $\sim 0.6 \mathrm{Gyr}$ after the merging event (Tomita et al. 1996). The SFR of galaxies would therefore be increased when they are located in the collision region of a cluster in the very central phases of merging $(\lessgtr 0.5 \mathrm{Myr}$ after the closest cores encounter). One has however to keep in mind that the observational results presented above are based on observations at different wavelengths (mostly optical and radio), that therefore trace SF episodes on different timescales. Homogeneous multi-wavelength studies of larger samples of clusters at different phases of merging are thus required to confirm the simple scenario about the effect of ram pressure on SF presented above.
In agreement with the results of our simulations, it has been suggested that the enhanced SF activity of the galaxies located in the collision region of merging clusters is due to dynamical ISM/ICM interactions (e.g. Poggianti et al. 2004; Ferrari et al. 2006). Actually, the ISM compression due to ICM ram pressure is most efficient where the density of the ICM and the relative velocity between the galaxy and the ICM reach their maximum, i.e. around the ICM compression bar originated by cluster mergers. Our numerical results show that, as expected, ram pressure is able to enhance the SFR of galaxies for several hundred Myr. In our idealised setup we do not find a significant drop in the SFR due to the depletion of the cold gas reservoir within our simulation time. However, in more realistic systems, where the galaxy encounters a density gradient and moves towards a denser cluster centre, we expect that the gas is consumed more rapidly and that the SFR drops on shorter timescales.

The dependence of SF on the dynamical state of clusters could be one of the key ingredients to explain the observed difference in the fraction of star-forming galaxies in similar redshift systems. Additionally, this dependence could shed more light on the origin both of the Butcher \& Oemler effect, and of the spectral evolution of the galaxies from higher to lower redshift: we know that in the hierarchical scenario of structure formation the merging rate between sub-clusters increases with redshift. If the net role of mergers is to trigger SF, this could be one of the possible explanations of the excess of blue galaxies detected a $z \sim$ 0.5 with respect to lower redshifts. Also the lower fraction of active cluster galaxies in the present Universe could be related to the lower fraction of merging clusters, and to the fact that member galaxies of local clusters have already experienced previous cluster interactions, being therefore gas-poor compared to their high- $z$ counterparts.

Ram pressure alone cannot explain the observed variation with density of the SFR in local cluster members. It has been proven (e.g. Balogh et al. 2004; Haines et al. 2007) that the equivalent width of the $\mathrm{H} \alpha$ emission line in star-forming galaxies does not depend strongly on the local density, while the fraction of star-forming objects does. This means that, in order to preserve the shape of the $\mathrm{EW}(\mathrm{H} \alpha)$ distribution, the physical mechanism(s) responsible for switching-off SF when a galaxy enter a cluster must act on short timescales $(<1 \mathrm{Gyr})$. Our simulations indicate that, in about $1 \mathrm{Gyr}$, ram-pressure stripping and galactic winds remove only $\sim 13 \%$ of the gas mass. This indicates that in nature additional processes are at work, which are not modelled in our simulations.

Based on our results (see top panel of Fig. 7), the existence of very extended $(\sim 100 \mathrm{kpc})$ tails of gas, possibly associated to intracluster star-forming regions (e.g. Gavazzi et al. 2001; Wang et al. 2004; Yoshida et al. 2004; Oosterloo \& van Gorkom 2005; Sun \& Vikhlinin 2005; Sun et al. 2006, 2007), cannot only be due to ram-pressure effects. They are possibly related to rampressure stripping of tidal tails and gaseous bridges created during galaxy-galaxy interactions (Kapferer et al. 2007b). The work presented in this paper is on the other hand able to explain the existence of extraplanar star-forming regions in several cluster late-type galaxies, that show evidence for ram-pressure stripping, but no extended tails of intracluster star-forming regions or clear signatures of interactions with other galaxies (e.g. Cortese et al. 2004; Kenney et al. 2004; Crowl et al. 2005; Cortese et al. 2007). Ram-pressure stripping acting on both isolated and interacting galaxies can finally be one of the possible physical mechanisms responsible for the formation of the observed intracluster stars (e.g. Freeman et al. 2000; Arnaboldi et al. 2003). 


\section{Summary and conclusions}

We have investigated the influence of ram-pressure stripping on the star formation and the resulting mass distribution in simulated spiral galaxies. We used a simplified model, where the ICM is distributed homogeneously and with a constant temperature, in order to isolate the effects caused by ram pressure.

- We found that ram-pressure stripping enhances the starformation rate by up to a factor of 3 over several hundred Myr. In total the mass of newly formed stars is about two times higher than in an isolated galaxy after $500 \mathrm{Myr}$ of ram pressure acting.

- The new stars are mainly formed in the central parts of the disc but a significant fraction forms also in the wake of the galaxy. As they are collisionless they do not feel the ram pressure and remain bound to the galaxy.

- Sub-structures form in the wake of the galaxy from compressed spiral arms of the original galaxy. These irregular shaped structures have sizes of roughly $1 \mathrm{kpc}$ in diameter and harbour newly formed stars. We term these structures "stripped baryonic dwarf" galaxies, as they do not possess a dark matter halo.

- The stripping radius, which is visible in the model galaxy is in good agreement with an analytical estimate using a Gunn \& Gott (1972) criterion.

- In a model galaxy, which moves edge-on through the ICM, the star formation rate is enhanced as well, however the distribution of the newly formed stars is different. The new stars are mainly formed in the central region of the disc.

- Observations of enhanced SF in merging galaxy clusters and of individual galaxies with tails of stripped gas support the results presented in this paper. As a galaxy moves through a real cluster environment, it encounters gradients in density, temperature, and the gravitational potential of the cluster and interactions with other galaxies. We will investigate this complex and degenerate interplay of the various processes in an upcoming paper.

Acknowledgements. We thank the anonymous referee for fruitful comments which improved the quality of the paper. The authors also thank Volker Springel for providing them with GADGET2 and his initial-conditions generator. Thomas Kronberger is a recipient of a DOC fellowship of the Austrian Academy of Sciences. The authors further acknowledge the UniInfrastrukturprogramm des BMWF Forschungsprojekt Konsortium Hochleistungsrechnen, the ESO Mobilitätsstipendien des BMWF (Austria), the Austrian Science Foundation (FWF) through grants P18523-N16 and P19300-N16, and the Tiroler Wissenschaftsfonds (Gefördert aus Mitteln des vom Land Tirol eingerichteten Wissenschaftsfonds).

\section{References}

Abadi, M. G., Moore, B., \& Bower, R. G. 1999, MNRAS, 308, 947 Agertz, O., et al. 2007, MNRAS, 380, 963

Arnaboldi, M., Freeman, K. C., Okamura, S., et al. 2003, AJ, 125, 514

Balogh, M., Eke, V., Miller, C., et al. 2004, MNRAS, 348, 1355

Bardelli, S., Pisani, A., Ramella, M., Zucca, E., \& Zamorani, G. 1998, MNRAS, 300,589

Bardelli, S., Zucca, E., \& Baldi, A. 2001, MNRAS, 320, 387

Belsole, E., Sauvageot, J.-L., Pratt, G. W., \& Bourdin, H. 2005, A\&A, 430, 385

Bravo-Alfaro, H., Cayatte, V., van Gorkom, J. H., \& Balkovsky, C. 2000, AJ, 119,580

Bravo-Alfaro, H., Cayatte, V., van Gorkom, J. H., \& Balkowski, C. 2001, A\&A, 379,347

Butcher, H., \& Oemler, A. Jr, 1978, ApJ, 219, 18
Caldwell, N., Rose, J. A., Sharples, R. M., Ellis, R. S., \& Bower, R. G. 1993, AJ, 106,473

Cayatte, V., van Gorkom, J. H., Balkovsky, C., \& Kotanyi, C. 1990, AJ, 100, 604 Cortese, L., Gavazzi, G., Boselli, A., \& Iglesias-Paramo, J. 2004, A\&A, 416, 119 Cortese, L., Marcillac, D., Richard, J., et al. 2007, MNRAS, 376, 157

Crowl, H. H., Kenney, J. D. P., van Gorkom, J. H., \& Vollmer, B. 2005, AJ, 130, 65

Domainko, W., Mair, M., Kapferer, W., et al. 2006, A\&A, 452, 795

Dressler, A. 1987, in Nearly Normal Galaxies: From the Planck Time to the Present, ed. S. Faber (New York: Springer), 276

Dressler, A., Smail, I., Poggianti, B., et al. 1999, ApJS, 122, 51

Dwarakanath, K. S., \& Owen, F. N. 1999, AJ, 118, 625

Evrard, A. E. 1991, MNRAS, 248, 8P

Ferrari, C., Benoist, C., Maurogordato, S., Cappi, A., \& Slezak, E. 2005, A\&A, 430, 19

Ferrari, C., Hunstead, R. W., Feretti, L., Maurogordato, S., \& Schindler, S. 2006, A\&A, 457, 21

Freeman, K. C., Arnaboldi, M., Capaccioli, M., et al. 2000, ASPC, 197, 389 Fujita, Y., Takizawa, M., Nagashima, M., \& Enoki, M. 1999, PASJ, 51, L1

Gavazzi, G., Marcelin, M., Boselli, A., et al. 2001, A\&A, 377, 745

Gingold, R. A., \& Monaghan, J. J. 1977, MNRAS, 181, 375

Gunn, J. E., \& Gott, J. R. III 1972, ApJ, 176, 1

Haines, C. P., Gargiulo, A., La Barbera, F., et al. 2007, MNRAS, 381, 7

Jáchym, P., Palouš, J., Köppen, J., \& Combes, F. 2007, A\&A, 472, 5

Kapferer, W., Knapp, A., Schindler, S., Kimeswenger, S., \& van Kampen, E. 2005, A\&A, 438, 87

Kapferer, W., Kronberger, T., Schindler, S., Ziegler, B. L., \& Böhm, A. 2006a, A\&A, 446, 847

Kapferer, W., Ferrari, C., Domainko, W., et al. 2006b, A\&A, 447, 827

Kapferer, W., Kronberger, T., Weratschnig, J., et al. 2007a, A\&A, 466, 813

Kapferer, W., Kronberger, T., Ferrari, C., et al. 2007b, A\&A, submitted

Katz, N., Weinberg, D. H., \& Hernquist, L. 1996, ApJS, 105, 19

Kenney, J. D. P., van Gorkom, J. H., \& Vollmer, B. 2004, AJ, 127, 3361

Kennicutt, R. C. 1989, ApJ, 344, 685

Kronberger, T., Kapferer, W., Schindler, S., et al. 2006, A\&A, 458, 69

Kronberger, T., Kapferer, W., Schindler, S., \& Ziegler, B. L. 2007,

[arXiv:0707.2301]

Larson, R. B., Tinsley, B. M., \& Caldwell, C. N. 1980, ApJ, 237, 692

Lavery, R. J., \& Henry, J. P. 1988, ApJ, 330, 596

Lucy, L. B. 1977, AJ, 82, 1013

Martin, C. L. 1999, ApJ, 513, 156

Miller, N. A. 2005, AJ, 130, 2541

Miller, N. A., \& Owen, F. N. 2003, AJ, 125, 2427

Miller, N. A., Owen, F. N., Hill, J. M., et al. 2005, ApJ, 613, 841

Mo, H. J., Mao, S., \& White, S. D. M. 1998, MNRAS, 295, 319

Mori, M., \& Burkert, A. 2000, ApJ, 538, 559

Moore, B., Lake, G., \& Katz, N. 1998, ApJ, 495, 139

Oosterloo, T., \& van Gorkom, J. 2005, A\&A, 437, L19

Owen, F. N., Ledlow, M. J., Keel, W. C., Wang, Q. D., \& Morrison, G. E. 2005, AJ, 129, 31

Poggianti, B. M. 2004, in Proceedings of Baryons in Dark Matter Halos, Novigrad, Croatia, 5-9 Oct. 2004, ed. R. Dettmar, U. Klein, \& P. Salucci, Published by SISSA, Proceedings of Science, http://pos.sissa.it, 104.1

Poggianti, B. M., Bridges, T. J., Komiyama, Y., et al. 2004, ApJ, 601, 197

Quilis, V., Moore, B., \& Bower, R. 2000, Science, 288, 1617

Roediger, E., \& Hensler, G. 2005, A\&A, 433, 875

Roediger, E., \& Brüggen, M. 2006, MNRAS, 369, 567

Roediger, E., \& Brüggen, M. 2007, MNRAS, 765

Roediger, E., Brüggen, M., \& Hoeft, M. 2006, MNRAS, 371, 609

Sakelliou, I., \& Ponman, T. J. 2006, MNRAS, 367, 1409

Schindler, S., Kapferer, W., Domainko, W., et al. 2005, A\&A, 435, L25

Schulz, S., \& Struck, C. 2001, MNRAS, 328, 185

Springel, V. 2005, MNRAS, 364, 1105

Springel, V., \& Hernquist, L. 2003, MNRAS, 333, 649

Springel, V., Di Matteo, T., \& Hernquist, L. 2005, MNRAS, 361, 776

Sun, M., \& Vikhlinin, A. 2005, ApJ, 621, 718

Sun, M., Jones, C., Forman, W., et al. 2006, ApJ, 637, L81

Sun, M., Donahue, M., \& Voit, G. M. 2007, ApJ, submitted [arXiv/0706 . 1220]

Tomita, A., Nakamura, F. E., Takata, T., et al. 1996, AJ, 111, 42

Toniazzo, T., \& Schindler, S. 2001, MNRAS, 325, 509

Vollmer, B., Cayatte, V., Balkowski, C., \& Duschl, W. J. 2001, ApJ, 561, 708

Vollmer, B., Balkowski, C., Cayatte, V., van Driel, W., \& Huchtmeier, W. 2004, A\&A, 419, 35

Wang, Q. D., Owen, F., \& Ledlow, M. 2004, ApJ, 611, 821

Yoshida, M., Yagi, M., Okamura, S., et al. 2002, ApJ, 567, 118 